\title{
Modelling Spatial and Temporal Emission Properties of the Young Pulsar Wind Nebula Kes 75
}

\author{
C. van Rensburg, ${ }^{a, b}$ A. Kundu ${ }^{b}$ and C. Venter ${ }^{b, *}$ \\ ${ }^{a}$ University of Namibia, Department of Physics, Private Bag 13301, Windhoek, Namibia \\ ${ }^{b}$ Centre for Space Research, North-West University, Potchefstroom Campus, Private Bag X6001, \\ Potchefstroom, South Africa, 2520 \\ E-mail: anukundu02@yahoo.com, Christo.Venter@nwu.ac.za
}

The H.E.S.S. Collaboration has firmly detected gamma-ray emission from HESS J1846-029, which is spatially coincident with Kes 75 (G29.7-0.3), one of the youngest composite supernova remnants in the Galaxy. This remnant contains the nebula of PSR J1846-0258, a glitching young pulsar with a particularly high spin-down luminosity, that has manifested magnetar-like bursts in 2006. However, H.E.S.S. was not able to distinguish between shell and nebular emission. This source may also plausibly be associated with the HAWC-detected source 2HWC J1844-032. Recent Chandra observations revealed a relatively rapid expansion of the embedded pulsar wind nebula over the past two decades and an X-ray flux decrease of $10 \%$ in 7 years. We apply a multizone spatio-temporal pulsar wind nebula model to the morphological and spectral data over several epochs, and find reasonable fits to the broadband radiation spectrum, X-ray surface brightness profile, photon spectral index in the X-ray energy range and expansion rate. Such spectral and morphological fitting constrains the model parameters, and may aid in clarifying the nature of the gamma-ray emission.

$3^{\text {th }}$ International Cosmic Ray Conference (ICRC 2021)

July 12th - 23rd, 2021

Online - Berlin, Germany

\footnotetext{
*Presenter
} 


\section{Introduction}

Being one of the youngest supernova remnants (SNRs) in the Galaxy, it is also a prototypical composite SNR that contains a pulsar wind nebula (PWN) powered by pulsar PSR J1846-0258. This pulsar is one of the most energetic known, with a period of $\sim 324 \mathrm{~ms}$, spin-down luminosity of $8.2 \times 10^{36} \mathrm{erg} \mathrm{s}^{-1}$ and a small characteristic age of $\sim 720 \mathrm{yr}$ [8]. In 2006, the braking index of PSR J1846-0258 was found to be $2.65 \pm 0.01$ [10]. It has recently exhibited magnetar-like outbursts $[4,9]$, with a subsequent softening of the X-ray spectrum [4] and a new braking index value of $2.16 \pm 0.13$ [9]. Kes 75 has also been detected at very-high energies (VHEs) by the High Energy Stereoscopic System (H.E.S.S.), with a flux of $2.4 \times 10^{-12} \mathrm{erg} \mathrm{cm}^{-2} \mathrm{~s}^{-1}$ and a spectral index $\Gamma \sim 2.3$ [16]. At these VHE energies the source is a point source, and it is not clear what the contribution of the SNR shell vs. that of the PWN might be. However, in the X-ray regime, the Chandra X-ray space telescope was able to observe the morphology of the source, as well as the expansion of the source from 2006 to 2016 [13].

In this proceedings article, we exploit the combination of spectral and spatial data on Kes 75 to constrain certain physical parameters. We use a leptonic emission code which calculates relativistic particle injection, transport, and emission as they traverse a PWN [19]. The code is able to yield both spatial and spectral information for the source in question. A detailed discussion of the code is given in Section 2, with the results and conclusions following in Section 3 and 4.

\section{The Model}

Here we briefly summarise the code we used. For full details, see van Rensburg et al. [19]. We solve the following transport equation:

$$
\begin{aligned}
\frac{\partial N_{\mathrm{e}}}{\partial t}= & -\mathbf{V} \cdot\left(\nabla N_{\mathrm{e}}\right)+\kappa \nabla^{2} N_{\mathrm{e}}+\frac{1}{3}(\nabla \cdot \mathbf{V})\left(\left[\frac{\partial N_{\mathrm{e}}}{\partial \ln E_{\mathrm{e}}}\right]-2 N_{\mathrm{e}}\right) \\
& +\frac{\partial}{\partial E}\left(\dot{E}_{\mathrm{e}, \mathrm{rad}} N_{\mathrm{e}}\right)+Q\left(\mathbf{r}, E_{\mathrm{e}}, t\right),
\end{aligned}
$$

with $N_{\mathrm{e}}\left(\mathbf{r}, E_{\mathrm{e}}, t\right)$ the number of leptons per unit energy and volume, $\mathbf{V}$ the bulk motion of leptons, $\kappa$ the spatially-independent diffusion coefficient, $\dot{E}_{\text {e,rad }}$ the total, i.e., synchrotron radiation (SR) and inverse Compton (IC) scattering, energy loss rates and $Q$ the particle injection spectrum. Here, $r$ is the radial dimension (assuming spherical symmetry) and $t$ the time since the PWN's birth. We solve this transport equation including one spatial dimension.

The particle injection spectrum is assumed to be a broken power law:

$$
Q\left(E_{\mathrm{e}}, t\right)= \begin{cases}Q_{0}(t)\left(\frac{E_{\mathrm{e}}}{E_{\mathrm{b}}}\right)^{-\alpha_{1}} & E_{\mathrm{e}, \min } \leq E_{\mathrm{e}}<E_{\mathrm{b}} \\ Q_{0}(t)\left(\frac{E_{\mathrm{e}}}{E_{\mathrm{b}}}\right)^{-\alpha_{2}} & E_{\mathrm{b}}<E_{\mathrm{e}} \leq E_{\mathrm{e}, \max }\end{cases}
$$

with $Q_{0}(t)$ the time-dependent normalisation constant that is determined by equating the first moment of the injection spectrum to a constant fraction $\eta$ of the time-dependent pulsar spin-down luminosity, $E_{\mathrm{e}}$ the particle energy, $E_{\mathrm{b}}$ the break energy and $\alpha_{1}$ and $\alpha_{2}$ the spectral indices. To limit the number of free parameters in this model, we assume that $\alpha_{1}$ and $\alpha_{2}$ are time-independent. 
We chose to parametrise the bulk flow of the leptons as well as the magnetic field profile. The first is parametrised according to $V(r)=V_{0}\left(r / r_{0}\right)^{\alpha_{\mathrm{V}}}$, with $V_{0}$ the bulk-flow normalisation, $r_{0}$ the inner (termination shock) radius of the PWN and $\alpha_{\mathrm{V}}$ the bulk-flow index parameter. We parametrise the magnetic field as $B(r, t)=B_{\text {age }}\left(r / r_{0}\right)^{\alpha_{\mathrm{B}}}\left(t / t_{\text {age }}\right)^{\beta_{\mathrm{B}}}$, with $B_{\text {age }}$ the present-day magnetic field at $r=r_{0}$ and $t=t_{\text {age }}$ the PWN age. With Kennel \& Coroniti [7], we assume that the magnetic field is toroidal and the bulk flow is purely radial. We also assume that, since the nebular plasma is a good conductor, we can apply ideal magnetohydrodynamic (MHD) equations (characterised by infinite macroscopic conductivity) to describe the PWN wind. In this case, Ohm's law becomes

$$
\mathbf{E}=-\frac{\mathbf{v}}{c} \times \mathbf{B}
$$

and by combining this with Faraday's law, we find [e.g., 3]

$$
\frac{\partial \mathbf{B}}{\partial t}=\nabla \times(\mathbf{v} \times \mathbf{B}) .
$$

To link the radial profiles of the magnetic field and bulk motion of the particles, we assume with $[6,11,12,15]$ that the temporal change in the magnetic field is slow enough that we can set $\partial \mathbf{B} / \partial t \simeq 0$ in the above equation, even for a time-dependent prescription of $B$. (This assumption holds exactly for steady-state models such as those of [7, 18].) From this follows $V B r=V_{0} B_{0} r_{0}=$ constant, which for our parametric specifications of the magnetic field and bulk flow implies that

$$
\alpha_{V}+\alpha_{B}=-1
$$

This relation is used to reduce the number of free parameters by one and to simplify our search for best-fit parameters. We next assume Bohm-type diffusion: $\kappa\left(E_{\mathrm{e}}\right)=\kappa_{B} E_{\mathrm{e}} / B(r, t)$, with $\kappa_{B}=c / 3 e$, $e$ denoting the elementary charge. Alternatively, we also consider a coefficient of the form $\kappa\left(E_{\mathrm{e}}\right)=$ $\kappa_{X}\left(E_{\mathrm{e}} / E_{0}\right)^{\delta}$, similar to what is used in Galactic cosmic-ray studies.

By solving Equation (1) numerically, we are able to predict the following four PWN properties in a spherically-symmetric scenario: (1) the spectral energy density (SED); (2) radiation spectra are calculated for each zone, which in turn are used to perform a line-of-sight (LOS) calculation that yields the predicted surface-brightness $(S B)$ profile at any snapshot in time; this allows us to furthermore determine the (3) expansion of the source for different epochs; (4) the SR component of the emitted SED for each zone is used to calculate the X-ray photon index for each epoch vs. radial distance from the embedded pulsar by fitting a power-law curve to the model SED in the $2.0-10.0 \mathrm{keV}$ energy band. All four these model predictions are fitted simultaneously to the data and the best-fit parameters are given in the table below.

\section{Results}

In this section, we describe our results for the best fits from the PWN model for the case of Kes 75 , for four different data sets and three different epochs. We consider a standard model, as described in [19], labelling it as old model. In what we term as new model, we have modified a few model parameters. Most importantly, to simulate the release of energy into the system as the pulsar manifests bursts, we increased bulk motion of the particles to 10 times its previous value, but 


\begin{tabular}{|c|c|c|c|c|}
\hline Fixed parameters & Old model & & New model & \\
\hline Pulsar period $(P)(\mathrm{s})$ & 0.324 & & 0.324 & \\
\hline Time-derivative of period $(\dot{P})\left(\mathrm{s} \mathrm{s}^{-1}\right)$ & $7.1 \times 10^{-12}$ & & $7.1 \times 10^{-12}$ & \\
\hline Spin-down luminosity $\left(L_{\text {age }}\right)(\mathrm{erg} / \mathrm{s})$ & $8.2 \times 10^{36}$ & & $8.2 \times 10^{36}$ & \\
\hline Braking index $(n)$ & 2.16 & & 2.16 & \\
\hline Distance to the source $(\mathrm{kpc})$ & 6.0 & & 6.0 & \\
\hline Index of the injected spectrum $\left(\alpha_{1}\right)$ & 1.4 & & 1.4 & \\
\hline Index of the injected spectrum $\left(\alpha_{2}\right)$ & 2.3 & & 2.3 & \\
\hline Break energy $\left(\gamma_{\mathrm{b}}\right)$ & $2.0 \times 10^{5}$ & & $6.0 \times 10^{5}$ & \\
\hline Magnetic energy conversion efficiency $(\eta)$ & 0.01 & & 0.01 & \\
\hline Particle energy conversion efficiency $(\epsilon)$ & 0.99 & & 0.99 & \\
\hline Sigma parameter $(\sigma)$ & 0.01 & & 0.01 & \\
\hline Magnetic field time dependence $\left(\beta_{\mathrm{B}}\right)$ & -1.0 & & -1.0 & \\
\hline Soft-photon components: & $T(\mathrm{~K})$ & $u\left(\mathrm{eV} / \mathrm{cm}^{3}\right)$ & $T(\mathrm{~K})$ & $u\left(\mathrm{eV} / \mathrm{cm}^{3}\right)$ \\
\hline Cosmic microwave background (CMB) & 2.76 & 0.23 & 2.76 & 0.23 \\
\hline Infrared & 25.0 & 2.5 & 15.0 & 0.8 \\
\hline Optical & 5000 & 1.4 & 5000 & 1.4 \\
\hline Fitted parameters & Old model & & New model & \\
\hline Radial parameter of the magnetic field $\left(\alpha_{\mathrm{B}}\right)$ & 0.0 & & 1.0 & \\
\hline Present-day magnetic field $(\mu \mathrm{G})$ & 11 & & 205 & \\
\hline Bulk flow normalisation $\left(10^{10} \mathrm{~cm} \mathrm{~s}^{-1}\right)$ & 0.012 & & 0.012 & \\
\hline Age (yrs) & 700 & & 700 & \\
\hline Diffusion coefficient normalisation $\left(\kappa_{\mathrm{X}} / 10^{27} \mathrm{~cm}^{2} \mathrm{~s}^{-1}\right)$ & Bohm & & 2 & \\
\hline Diffusion coefficient exponent $(\delta)$ & 1.0 & & 0.3 & \\
\hline
\end{tabular}

Table 1: Best-fit parameters for PWN Kes75 using old and new models (see text for differences).

for the last 50 years of the current age of the PWN. Thus, we consider the changes only in last few decades of the current lifetime of the PWN. We also use the second parametrisation of the diffusion coefficient. We summarise the difference in model parameters obtained during the fitting process for the two models in Table 1. The results of our fits to four data sets are shown in Figure 1 to Figure 4.

A multi-wavelength fit to the SED for Kes 75 is shown in Figure 1. The fits are shown for the observational data taken from [17] (radio: [2, 14]; X-rays: [5]; TeV: [1].) While the old model provides a reasonable spectral fit, mostly to the radio and X-ray data, the new model yields an improved overall fit.

We next show the SB for Kes 75, for both models, in Figure 2. The SB is normalized to 1 around $10^{\prime}$, and is plotted against distance (in arcsec) from the centre of the PWN along the $x$-axis. Both models follow the trend where brightness drops off as we move away from the centre of the PWN. The data points in blue are taken from the study by [13].

In Figure 3 the change in the X-ray index is shown for different epochs. This change is rather small, as measured by [13] at four different years, 2000, 2006, 2009 and 2016. During this time, the predicted X-ray spectrum does not change significantly, as this represents only a short final period in the overall temporal evolution of the source. Still, it is a good test to see whether the index and the flux of the model and the source are comparable. Both Figure 2 and 3 are closely linked to the 


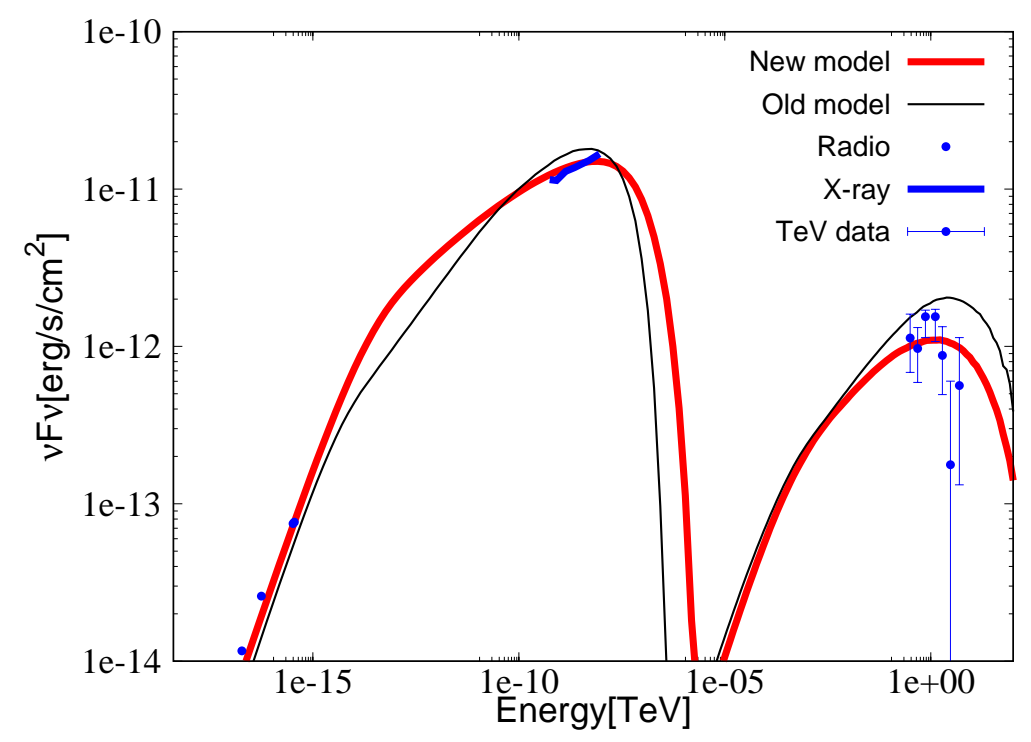

Figure 1: SED for Kes 75 shown for old (in black) and new (in red) model. Radio, X-ray, and VHE observational data are taken from [17] (see text for more details).

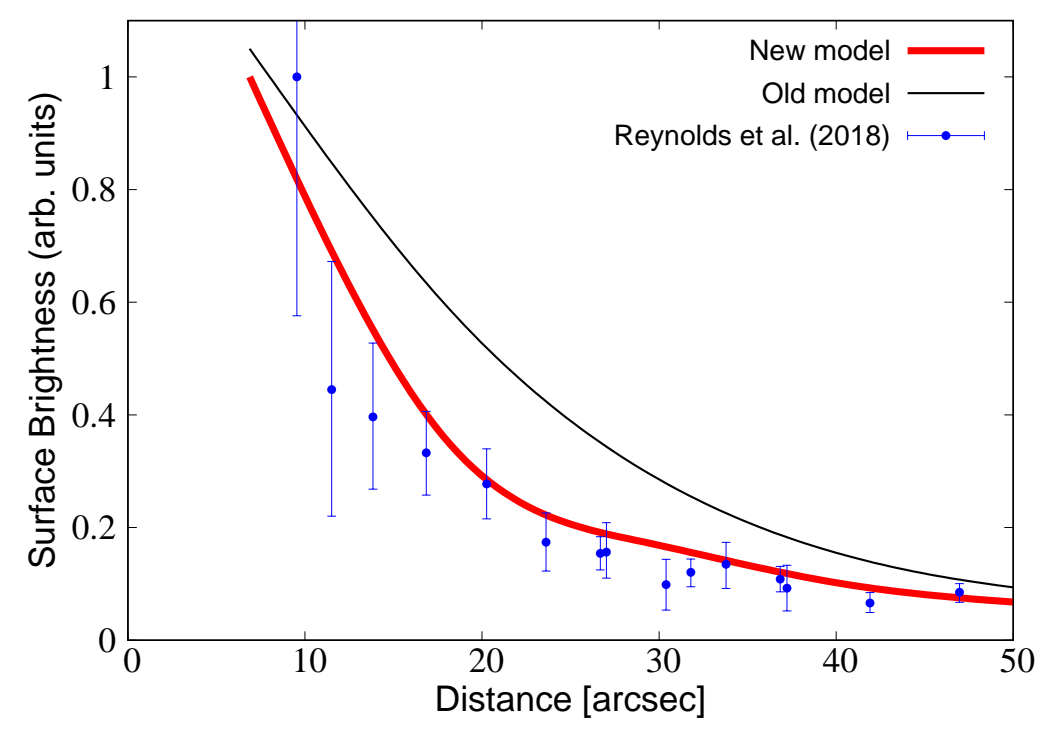

Figure 2: SB for Kes 75 for old (in black) and new (in red) model with respect to distance from the centre of the PWN (in arcsec.) The measured SB profile from [13] is shown as blue data points. 


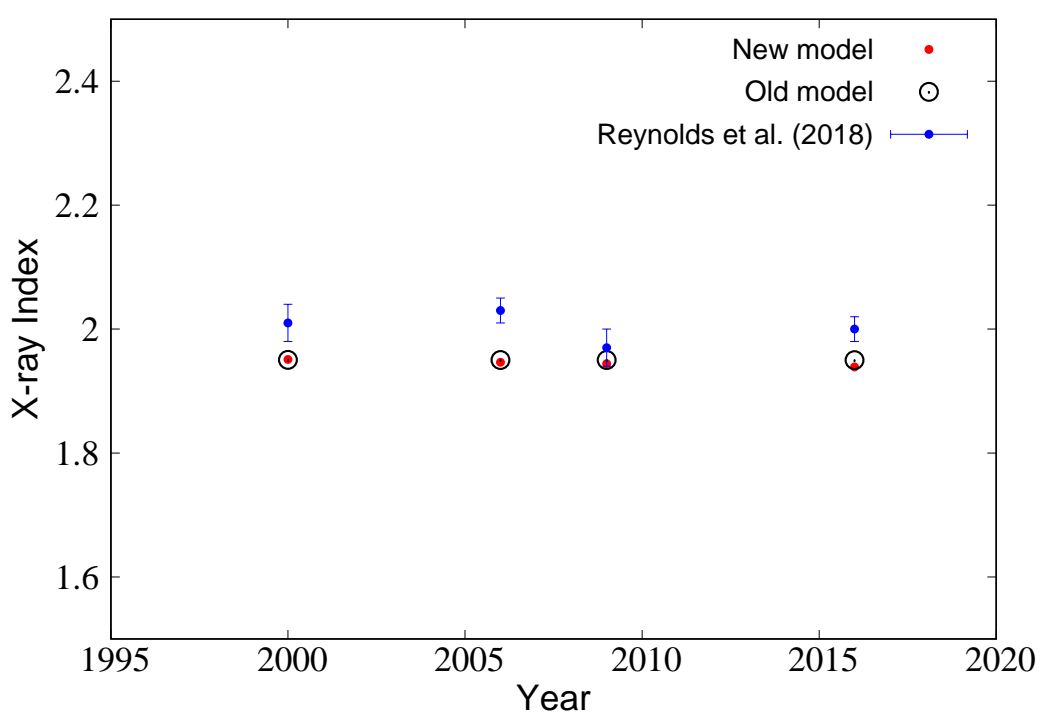

Figure 3: X-ray photon indices for Kes 75 shown for old (in black) and new (in red) model, spanning between the year 2000 to 2016. Inferred values by [13] for the corresponding years are also shown in blue.

SED in Figure 1. Thus, a good fit to the SED is a good start; however, the spatial data represent further avenues for constraining the model best-fit parameters.

Finally, Figure 4 indicates the expansion of the PWN at three different epochs for both model cases, including measurements by [13]. These epochs are indicated by 1, 2, and 3 for the periods 2000-2016, 2006-2016, and 2009-2016 respectively. Our new model fits the data set of [13] better than the old model for all three epochs. There may still be more improvements needed to obtain a yet superior fit to the data.

\section{Conclusions}

This proceedings article presents our first fitting results to spectral and new spatial data of Kes 75. As indicated in the figures above, we obtained improved fits to all four features (SED, SB profile, spectral index vs. $r$, and expansion,) using our new model. A major feature of this model is a recent abrupt increase in bulk flow, something that might be explained via energy deposition into the system by the bursting behaviour of the central pulsar. The SB is also particularly sensitive to the assumed diffusion coefficient, while the SED shape (particularly the SR component) depends on the assumed present-day nebular field strength.

Since there are a number of free parameters in the model, some of which may be degenerate, we believe that further study of the said features with our spatio-temporal code could improve our fitting results. We plan to continue to refine the parameters to obtain an even better fit to Kes 75 (Van Rensburg et al., in prep.). We will also study the effect of different changes (separately and in combination) to the underlying physics description in the model on the four observables presented in this work. In this way, we want to optimally exploit the constraining power of the 


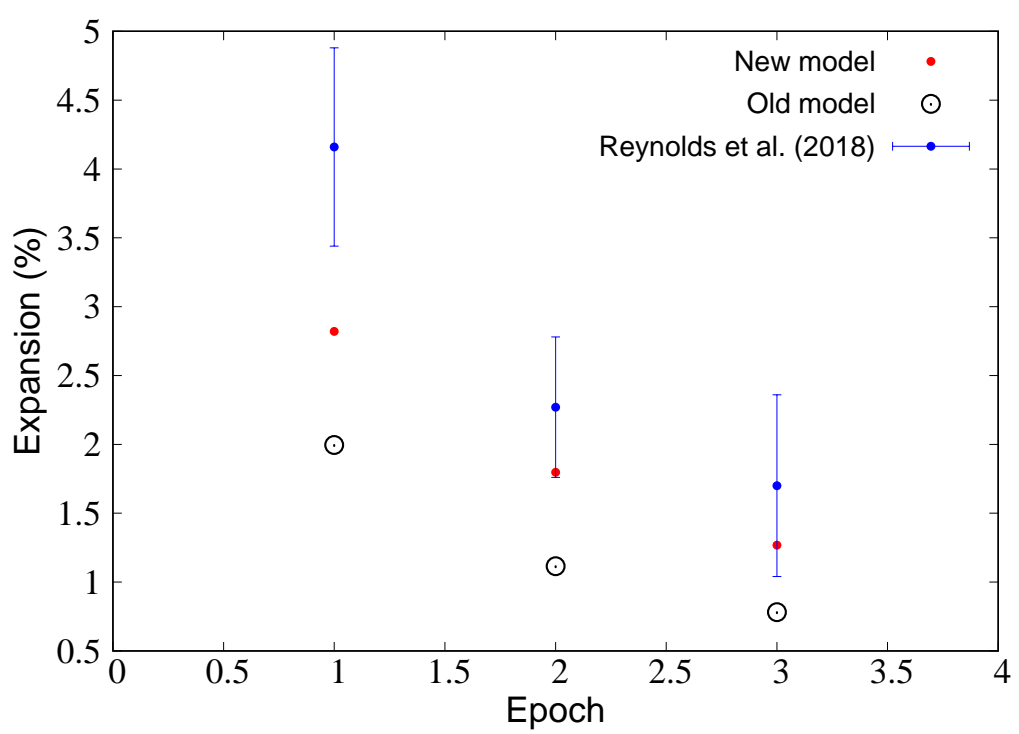

Figure 4: Expansion of Kes 75 for three different epochs (1: 2000-2016, 2: 2006-2016 and 3: 2009-2016), for old (in black) and new (in red) model. Values fitted by [13] for the corresponding epochs are shown in blue.

combination of spectral and spatial PWN data. Once we better understand such constraints, we can fine-tune the code for application to a sample of PWNe that have both spectral and spatial data available. Application to such a sample may lead to the revision of $B$-field and bulk motion profile parametrisations (calculations) in the face of new, independent dynamical or MHD calculations, the linking of these two profiles and the effect of burst-like (or glitching) behaviour from the pulsar on temporal injection properties of the system.

\section{Acknowledgements}

This work is based on the research supported wholly / in part by the National Research Foundation (NRF) of South Africa (Grant Numbers 92860, 93278, and 99072). The Grantholder acknowledges that opinions, findings and conclusions or recommendations expressed in any publication generated by the NRF supported research is that of the author(s), and that the NRF accepts no liability whatsoever in this regard. We also acknowledge support from the UKRI STFC Global Challenges Research Fund project ST/S002952/1.

\section{References}

[1] Acero F., et al., 2013, ApJ, 773, 77

[2] Bock D. C. J., Gaensler B. M., 2005, ApJ, 626, 343

[3] Ferreira S. E. S., de Jager O. C., 2008, A\&AS, 478, 17 
[4] Gavriil F. P., Gonzalez M. E., Gotthelf E. V., Kaspi V. M., Livingstone M. A., Woods P. M., 2008, Science, 319, 1802

[5] Helfand D. J., Collins B. F., Gotthelf E. V., 2003, ApJ, 582, 783

[6] Holler M., Schöck F. M., Eger P., Kießling D., Valerius K., Stegmann C., 2012, A\&AS, 539, A24

[7] Kennel C. F., Coroniti F. V., 1984, ApJ, 283, 694

[8] Kuiper L., Hermsen W., 2009, A\&AS, 501, 1031

[9] Kumar H. S., Safi-Harb S., 2008, ApJ, 678, L43

[10] Livingstone M. A., Kaspi V. M., 2006, in AAS High Energy Astrophysics Division \#9. p. 1.21

[11] Lu F.-W., Gao Q.-G., Zhu B.-T., Zhang L., 2017, MNRAS, 472, 2926

[12] Lu F.-W., Gao Q.-G., Zhu B.-T., Zhang L., 2019, A\&AS, 624, A144

[13] Reynolds S. P., Borkowski K. J., Gwynne P. H., 2018, ApJ, 856, 133

[14] Salter C. J., Reynolds S. P., Hogg D. E., Payne J. M., Rhodes P. J., 1989, ApJ, 338, 171

[15] Schöck F. M., Büsching I., de Jager O. C., Eger P., Vorster M. J., 2010, A\&AS, 515, A109

[16] Terrier R., Djannati-Atai A., Hoppe S., Marand on V., Renaud M., de Jager O., 2008, in Aharonian F. A., Hofmann W., Rieger F., eds, American Institute of Physics Conference Series Vol. 1085, American Institute of Physics Conference Series. pp 316-319, doi:10.1063/1.3076670

[17] Torres D. F., Cillis A., Martín J., de Oña Wilhelmi E., 2014, J. HEAP, 1, 31

[18] Vorster M. J., Tibolla O., Ferreira S. E. S., Kaufmann S., 2013, preprint, (arXiv: 1308. 1626)

[19] van Rensburg C., Krüger P. P., Venter C., 2018, MNRAS, 477, 3853 